\title{
Assessment of Blade Strength for Small Wind Turbine Applications
}

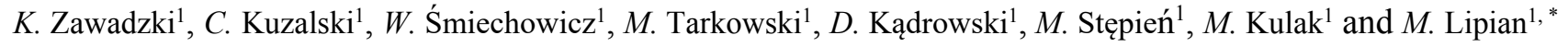 \\ ${ }^{1}$ Institute of Turbomachinery, Lodz University of Technology
}

\begin{abstract}
Small Wind Turbines (SWTs) are an increasingly developing Renewable Energy Source, thanks to the gradual popularisation of the prosumer energy generation approach. This, however, requires maximum safety of the machine, working next to households. Reliability is also necessary from the financial point of view, as SWTs should withstand 20-30 years of operation without any serious failure to ensure investment profitability. The weakest SWT element is arguably its most exposed one - the rotor - hence the important role played by the blade strength tests. The experimental analysis becomes preferable with increasing popularity of new materials (composites) and production techniques (additive manufacturing). This paper sets out to develop a test stand for static strength examination of 3D-printed SWT blades. This kind of objects is often considered as more vulnerable to structural failures than those created via machining techniques, due to their anisotropic structure, so ensuring structural integrity is crucial for safety reasons.
\end{abstract}

\section{Introduction}

Wind turbines are modern devices that convert kinetic energy of the wind into electrical energy [1]. Wind affects the wear of every part of a wind turbine. Blades are the most vulnerable to destruction components because they always have to face wind in order to transmit its power into the torque [2]. Potentially dangerous forces acting on a wind turbine blade appear not only during each sudden gust, but also during a constant, uniform wind [1]. Static strength is therefore a key parameter of every wind turbine blade [3]. However, blades are often made from composites and anisotropic materials, making their construction relatively complex. Therefore, certain safety factor must be considered when performing any numerical and analytic studies. This is the main reason for which static tests are of a fundamental importance to determine the strength of wind turbine blades in a practical way [3].

\subsection{Experimental studies of wind turbine blades strength}

In order to properly carry out blade strength tests, it is essential to firstly define the main forces acting on it. According to Tawade et al., the most significant static forces acting on a horizontal axis wind turbine (HAWT) blades are: thrust, tangential, gravitational and centrifugal forces, coming mostly from aerodynamics and rotation [4]. The centrifugal force produces tensional stresses, while thrust and tangential loads cause bending in edgewise and flapwise directions [4].
Blades tend to bend more in the latter direction than in the former one (Branner et al.) [5]. Hence, the biggest deflections and stresses occur in the flapwise direction of bending (shear and torsion) [6], which was described by Paquette and Veers as the best to approximate extreme loads from the wind [7]. To emulate the flapwise bending, a whiffletree mechanism can be used [7]. The blade can be considered as a cantilever during the experiment [4]. This approach allows to apply a rigid support at the root and a system of forces (for example a whiffletree) to the blade surface. The way to optimize the shape of a whiffletree was described by Yeniceli [8]. By putting distributed, discrete loads on a tested specimen, it is possible to obtain load distribution almost identical to real one [8].

Static bending tests were also performed at the Lodz University of Technology. The main purpose of the experiment was to develop a method for the stress-deflection curve determination for a $3 \mathrm{D}$ printed blade. The whiffletree mechanism was used in order to determine strength and Young modulus of the blade. The $160 \mathrm{~mm}$-long blade of variable chord length and twist angle is based on SG6040 aerofoil family. First, the CAD model of a blade was designed. Later it was printed in the 3D printer using the ABS plastic [9], thus its properties are anisotropic. The anisotropy comes from the fact that the model consists of layers of plastic that were applied in parallel one after the other, thus the blade is not made of an uniform material. Layers should not be printed in the radial direction, so as to avoid the destructive action of centrifugal forces.

Corresponding author: michal.lipian@p.lodz.pl 


\section{Methodology}

Using the blade element momentum theory, aerodynamic forces per unit span were calculated as a first step of the blade strength investigation. Since the aerodynamic forces are with respect to the relative velocity direction changing in each section, they should be defined in a global coordinate system. After calculation of the aerodynamic loads, internal forces were obtained. Spanwise distribution of the shear forces ( $\mathrm{X}$ and $\mathrm{Z}$ direction), pitching moment $\left(\mathrm{M}_{\mathrm{y}}\right)$ and bending moments $\left(\mathrm{M}_{\mathrm{x}}\right.$ and $\left.\mathrm{M}_{\mathrm{z}}\right)$ were determined, as shown in Figure 1.
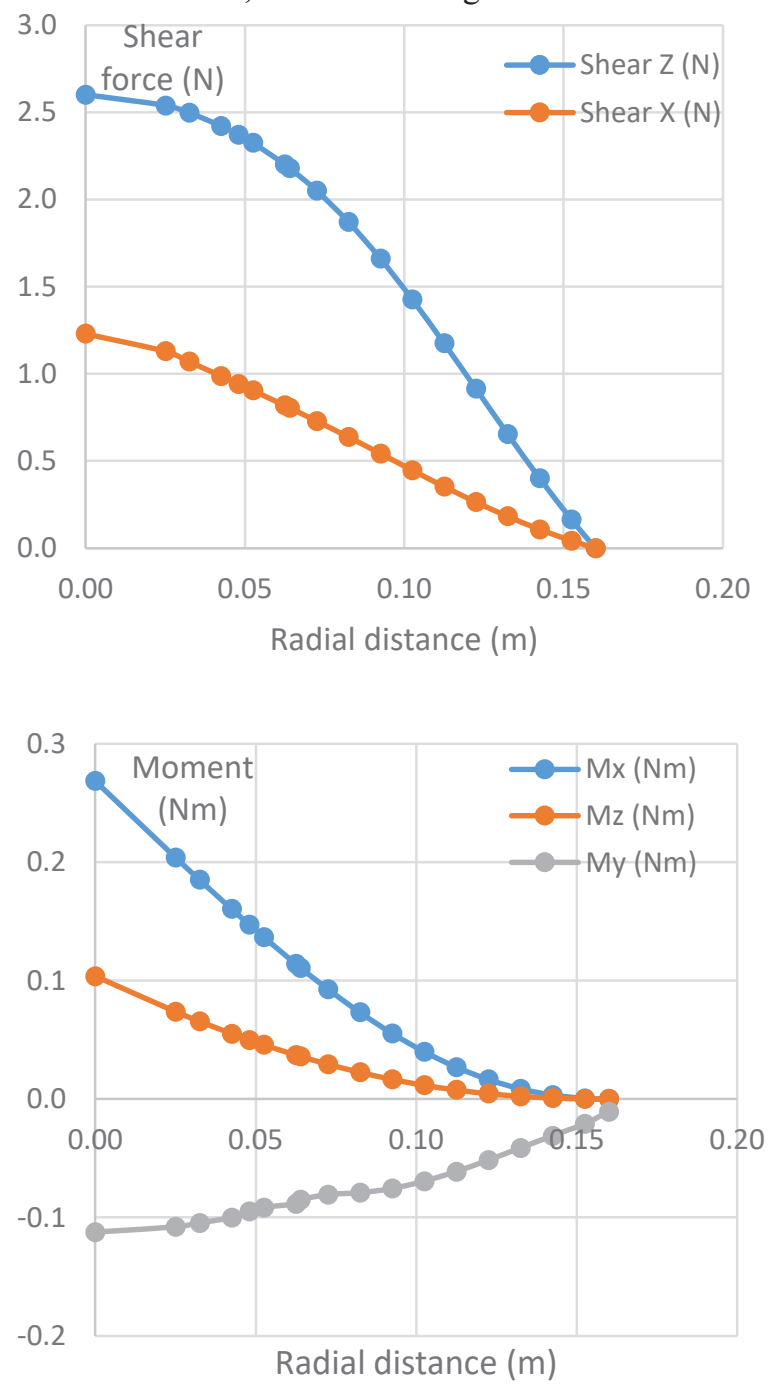

Figure 1. Results of calculation of forces acting on a blade

It can be observed that shear force in $\mathrm{Z}$ direction and bending moment about $\mathrm{X}$-axis are the most critical loads among the analysed ones. Therefore, these forces are considered as the design loads for further considerations and design of the whiffletree.

\subsection{Test stand preparation}

The first step in the strength optimisation of a blade is the static test. It was performed using a four-saddle point whiffletree as presented in Figure 2. The aim was to create a load distribution almost identical to the load distribution determined earlier and described in the previous section.

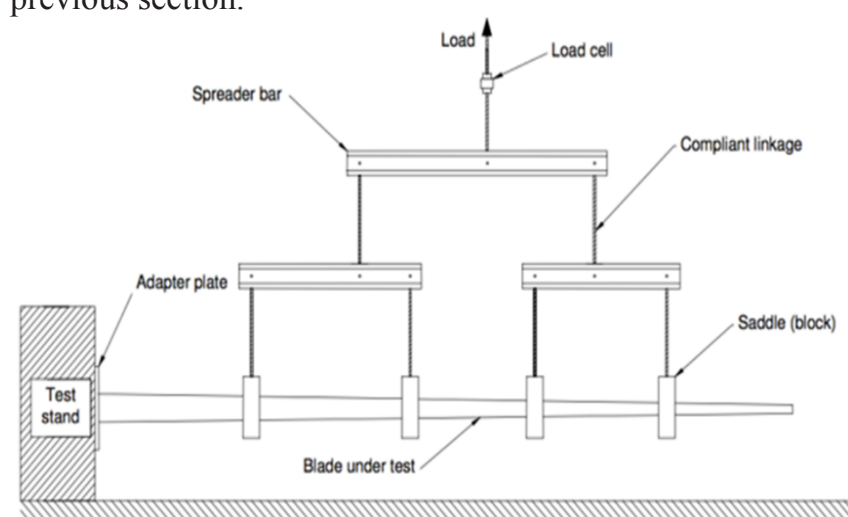

Figure 2. Scheme of a whiffletree [8]

Optimisation of the whiffletree allows to determine its final design and requires consideration of eight parameters: the positions and the percentage of the total load for each of the four saddles. The optimisation is automatized using the Matlab code, elaborated by Yeniceli [8].

\section{Static test}

The principle of the static test is to see the deflection of the blade under the load and to see how much load can be applied before the blade rupture. To assess the deflection, a graph paper was put behind the blade (see Figure 3) and a camera captured the deflection under different applied loads. The displacement was assessed at the blade tip and three points of fixation of nylon chords ("saddles"; the one closest to the adapter plate was omitted, as the deflection was too small for a credible measurement).

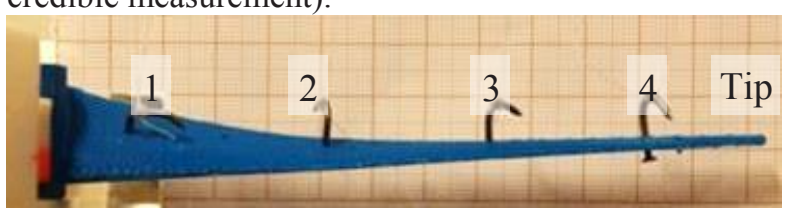

Figure 3. 3D printed blade subjected to the test stand

\subsection{Test results}

Raw results of the deflection analysis are presented in Figure 4. According to the Matlab optimisation [8], the theoretical load (mass), which reproduces real load distribution during windy conditions, is equal to 350 grams. After reaching and exceeding this value during the test, not only the blade did not break, but it still remained in the elastic region of deflection. Measurements were continued until reaching the mass of 3425 grams, when the nylon linkage (rope) broke. Even in this case the blade went back to its original geometry and no sign of plastic deformation was found. 


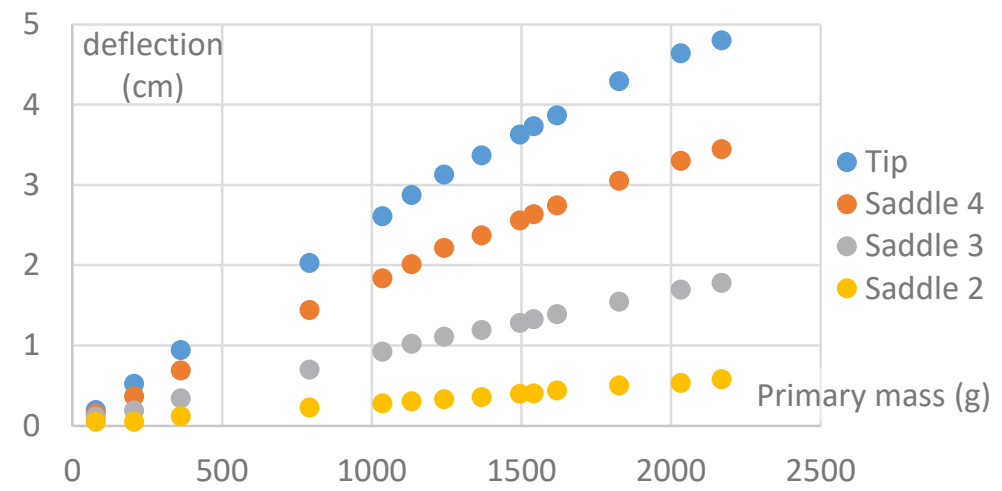

Figure 4. Results of deflection analysis of the $3 \mathrm{D}$ printed blade

The final objective of the static test is to have a link between deflection and stress. Because the deflection of the first saddle was not measured and the torque in the last saddle is equal to zero, only the evolution of the deflection of the saddle 2 and 3 (Figure 5) can be plotted

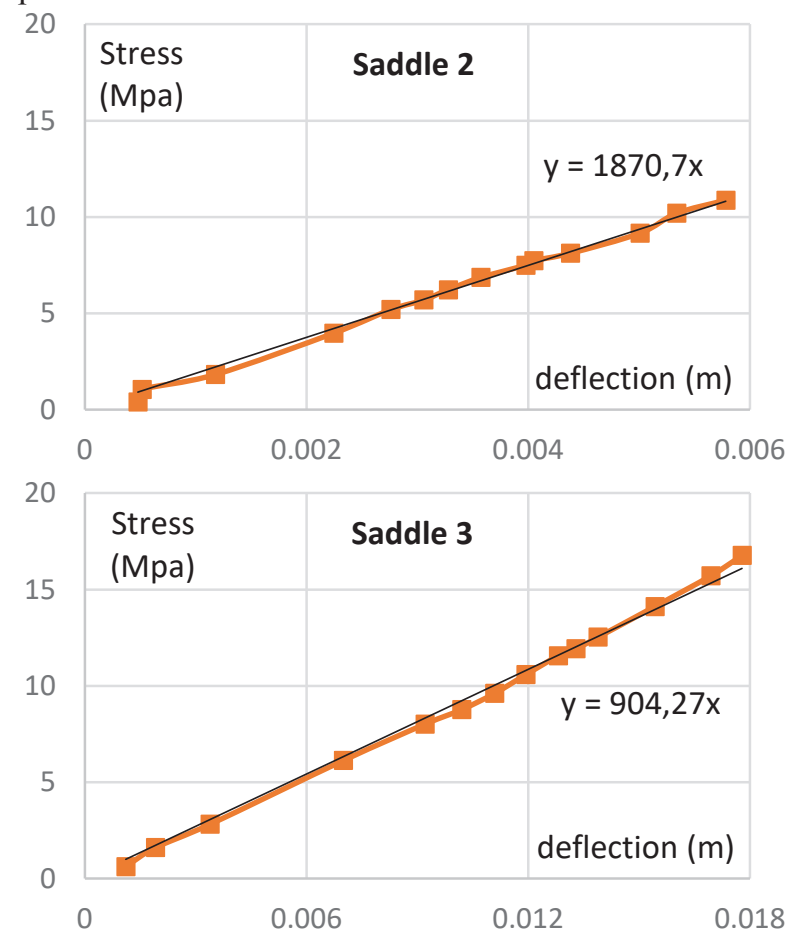

Figure 5. Relationship between applied stress and deflection of saddle 2 and 3

Those two graphs are however not enough to fully define the stress-deflection curve of the blade. To find a link between the deflection and the stress, Young's modulus $\mathrm{E}$ of the anisotropic blade should be found. The starting point for this analysis is the equation (1),

$$
w(x)^{\prime \prime}=\frac{M(x)}{E I_{x}},[\mathrm{SI}]
$$

where $\mathrm{w}(\mathrm{x})$ is the deflection, $\mathrm{M}$ is the torque, $\mathrm{E}$ the Young's modulus and $\mathrm{I}_{\mathrm{x}}$ the second moment of area. In the studied case the second moment of area and the torque are not constant along the blade, so they were calculated for 17 consecutive blade sections. Hence, it was possible to obtain the function of the $\mathrm{M} / \mathrm{I}$ ratio evolution with the position along the blade. Using curves from Figure 4 one can obtain deflection functions $\mathrm{w}(\mathrm{x})$ and differentiate them two times. The last step is to divide values of ratio $\mathrm{M} / \mathrm{I}$ over values of the second derivative of deflection function w", according to the formula (2):

$$
\mathrm{E}=\frac{M}{\mathrm{w} \prime I_{x}},[\mathrm{SI}]
$$

The obtained values of Young's modulus along the blade are shown in Table 1.

Table 1. Averaged Young's modulus at specific radial distance of the blade

\begin{tabular}{cc}
\hline $\begin{array}{c}\text { Radial } \\
\text { distance } \\
{[\mathrm{m}]}\end{array}$ & $\begin{array}{c}\text { Average } \\
\text { Young's } \\
\text { Modulus } \\
{[\mathrm{GPa}]}\end{array}$ \\
\hline 0.11 & 1.447 \\
0.14 & 1.116 \\
0.16 & 1.091 \\
\hline
\end{tabular}

With the studied blade the radial distance starts at $0.025 \mathrm{~m}$ and ends at $0.16 \mathrm{~m}$, so the young modulus fluctuates between $1.09 \mathrm{GPa}$ and $1.45 \mathrm{GPa}$. This value is of similar order of magnitude as the one for a solid material, given by the filament manufacturer, that is $2.0 \mathrm{GPa}[10]$. It is also smaller than the latter, as would be expected from an anisotropic 3D print.

\section{Discussion}

The similarity of Young's modulus values obtained on the base of experiment with the value of Young's modulus provided by the manufacturer is a proof that results of the strength tests are reliable. Consequently, throughout the static study it was proven that $3 \mathrm{D}$ anisotropic printed blades are relatively (or even unexpectedly) tough. A load ten times bigger than the theoretical one (around $34 \mathrm{~N}$ ) was not enough to delaminate the model. The safety factor for the static test is almost equal to ten, which is more than enough in terms of use of wind turbine blades. Hence, 3D printed blades for a small wind turbine are a viable option. Furthermore, 3D printing technology provides an accurate and precise shape of a model. 
It should be noticed that tests were performed only for a scaled-down model of a blade. Satisfactory results allow to assume that similar strength results possibly could be achieved also for a model in a full scale. However, without making a separate strength test on full-scale blades, this theory cannot be considered universally true. Thus, the next step of the strength investigation of wind turbine wings should involve a test on big blades, using similar method developed in this study. By using the Matlab application, load calculation and the four-saddle whiffletree optimisation can be applied to any other blade. In order to perform a test more accurately and determine the Young modulus with a bigger precision, more than three points on the blade can be considered.

\section{Summary}

The described method for assessment of wind turbine blades proved that the essential mechanical parameters of the 3D-printed model can be determined in a traditional, relatively easy to execute way. Although finally the determination of the static strength of the blade proved to be impossible due to the fixation system rupture, the experiment of a small - scale blade turned out to be a sufficient proof that method involving the use of a whiffletree gives reliable results. With the use of basic equations and data of deflection from experiment it was possible to estimate the Young's modulus.

The authors of the manuscript believe that the presented technique may be scaled-up and a similar mechanism of testing can be used for full-scale SWT blades. For this sake, the next necessary actions include testing the technique's robustness in operation with different materials - composites, reinforced plastic, etc. The proposed testing mechanism is also an interesting option for didactic applications, in both strength of materials and aeroelastic phenomena analysis.

\section{References}

1. Schubel P J and Crossley R J 2012 Wind Turbine Blade Design Energies 5 pp 3425-49

2. Malhotra P 2014 Advanced Blade Testing Methods for Wind Turbines Master Thesis (Massachusetts, University of Massachusetts Amherst)

3. https://blaest.com/links-2/

4. Tawade S V 2014 Fatigue Life Optimization of Wind Turbine Blade International Journal of Research in Engineering and Technology vol 3 pp 843-50

5. Branner $\mathrm{K}$ and Berggreen $\mathrm{C}$, Berring $\mathrm{P}$, Knudsen H W 2007 Torsional Performance of Wind Turbine Blades - Part II: Numerical Validation Proc. $16^{\text {th }}$ Int. Conf. on Composite Materials (Kyoto, Japan)

6. Eder MA, Bitsche R, Nielsen M, Branner K 2014 A practical approach to fracture analysis at the trailing edge of wind turbine rotor blades Wind Energy vol 17 pp 483-97

7. Paquette $J$ and Veers Paul 2007 Increased Strength in Wind Turbine Blades Through Innovative Structural Design Test 3 (Albuquerque, NM, USA, Sandia National Laboratories)

8. Yeniceli S C 2014 Design Optimization of Whiffletree for Wind Turbine Blade Testing MSc Thesis (Ankara, Middle East Technical University)

9. Lipian M, Kulak M, Stępień M 2019 Fast Track Integration of Computational Methods with Experiments in Small Wind Turbine Development Energies 2019, 12 (Lodz, Poland, Institute of Turbomachinery)

10. https://github.com/superjamie/lazyweb/wiki/3DPrinting-Filament-Properties 